COLABORACIÓN ESPECIAL

\title{
ANÁLISIS DEL MODELO SALUTOGÉNICO EN ESPAÑA: APLICACIÓN EN SALUD PÚBLICA E IMPLICACIONES PARA EL MODELO DE ACTIVOS EN SALUD
}

\author{
Francisco Rivera de los Santos (1,2), Pilar Ramos Valverde (2), Carmen Moreno Rodrí- \\ guez (2) y Mariano Hernán García (3).
}

(1) Departamento de Psicología Clínica, Social y Experimental. Universidad de Huelva.

(2) Departamento de Psicología Evolutiva y de la Educación. Universidad de Sevilla.

(3) Escuela Andaluza de Salud Pública. Consejería de Salud. Junta de Andalucía.

\begin{abstract}
RESUMEN
Este artículo tiene el objetivo de tratar en profundidad uno de los modelos más revolucionarios e influyentes para la comprensión de las variables y procesos que explican la salud humana. A partir de una nueva visión en el análisis de las consecuencias del holocausto nazi, un médico-sociólogo -Aaron Antonovsky-, consiguió influir en la medicina y la ciencia del comportamiento, facilitando las claves para el óptimo desarrollo de la salud pública actual. A pesar de que esta teoría empezó a forjarse en los años 70 del siglo XX, su desarrollo real y su expansión están sucediendo en los últimos años. De hecho, en España existe muy poca literatura con base científica que analice con detalle las claves teóricas del modelo. Este trabajo trata de cubrir esa carencia y, para ello, y en primer lugar, expone cómo surgió el constructo salutogénesis, el contexto sociocultural que lo impulsó, así como la importancia que adquiere en la salud pública actual. Con este objetivo, en estas páginas se analizan las bases teóricas del modelo salutogénico, con especial énfasis en sus antecedentes y precursores, así como en sus inicios, desarrollo y expansión actual.
\end{abstract}

Palabras clave: Salutogénesis. Salud pública. Promoción de la Salud. Activos en salud. Bienestar.

\section{ABSTRACT}

\section{Salutogenic Model Analysis in Spain: Application in Public Health and Impli- cations for Asset Health Model}

This article seeks to provide an in depth review about one of the most revolutionary and influential methods used in understanding the variables and processes that explain human health. Based on a new vision in the analysis of the consequences of the Nazi Holocaust, a doctor-sociologist-Aaron Antonovskymanaged to influence medicine and behavioral science by facilitating the keys for the optimal development of public health today. Despite the fact that this theory began appearing in the 1970 s in the 20th century, its real development and expansion have been seen in recent years. In fact, in Spain, there is little scientific literature that analyses the theoretic keys of the model in depth. This work seek to cover this gap; to achieve this objective, it first presents how the construct of salutogenesis arose, the social-cultural context that promoted it, as well as the importance public health acquires today. This is the aim of this work, which analyses the theoretical bases of the salutogenesis model, with specific emphasis on its background and precursors, as well as its inception, development and current expansion.

Key words: Salutogenesis. Public Health. Health Promotion. Health Assets. Well-being. 


\section{INQUIETUDES DE UN MÉDICO- SOCIÓLOGO: NACIMIENTO DEL MODELO SALUTOGÉNICO}

Aaron Antonovsky nació en Estados Unidos en 1923 y sirvió en las tropas de este país durante la II Guerra Mundial. En 1960 emigró a Israel para trabajar en el Instituto Israeli para las Ciencias Sociales Aplicadas y en el Departamento de Medicina Social en la Universidad Hebrea de Jerusalén. En los años 70 comenzó a desarrollar el modelo salutogénico que, como se expone a continuación, influyó en el pensamiento de los médicos y científicos del comportamiento por su explicación de los factores que forman las bases de la salud humana.

En una de sus investigaciones empíricas más importantes, Antonovsky estudió a mujeres nacidas en Europa central entre 1914 y 1923, algunas de las cuales habían estado internadas en campos de concentración ${ }^{1}$. Tal y como se esperaba, el grupo de supervivientes de los campos de concentración exhibía significativamente más signos de enfermedad en comparación con las mujeres del grupo control. Sin embargo, hasta el 29\% de las antiguas prisioneras alegaron tener una relativamente buena salud mental, a pesar de sus experiencias traumáticas. Antonovsky se preguntó cómo estas mujeres lograron mantenerse saludables a pesar de la extrema presión que se ejerció sobre ellas. Al formular la pregunta en positivo, este cambio de perspectiva influyó en todas sus investigaciones posteriores. En concreto, a partir de esta investigación, Antonovsky utilizó tres preguntas que fueron claves en la fundación de su teoría:

- ¿Por qué las personas se mantenían saludables a pesar de estar expuestas a tantas influencias perjudiciales?

- ¿Cómo se las arreglan para recuperarse de enfermedades?

- ¿Qué tienen de especial las personas que no enferman a pesar de la tensión más extrema?
Antonovsky utilizó estas tres preguntas como el punto central de partida para sus investigaciones en salud. Dentro del marco de estas investigaciones, estas preguntas también se consideraron importantes a la hora de entender la capacidad y voluntad de los expatriados para hacer frente a las presiones recibidas durante las diversas fases de la emigración. En este sentido, es importante reflexionar cómo el paradigma salutogénico y la salud pública posmoderna paradójicamente nacen en las cenizas de las víctimas del Holocausto. En definitiva, a partir de las investigaciones centradas en una nueva forma de observar las consecuencias que los horrores de la guerra tuvo en las personas, el modelo salutogénico comienza a surgir, basándose en las siguientes asunciones ${ }^{2-3}$ :

- el énfasis se hace sobre los orígenes de la salud y el bienestar,

- la principal preocupación se centra en el mantenimiento y realce del bienestar, $y$

- la hipótesis de que los factores estresantes son intrínsecamente negativos es rechazada en favor de la posibilidad de que los factores estresantes puedan tener consecuencias saludables o beneficiosas, dependiendo de sus características y de la capacidad de las personas para resolverlos.

Antonovsky criticó el típico enfoque salud-enfermedad, basado en el modelo tradicional patogénico, en el que las condiciones de salud y enfermedad son por lo general mutuamente excluyentes. En su crítica hacia el tradicional enfoque occidental patogénico, Antonovsky propuso entender la salud como un continuo de salud-enfermedad. En este continuo identificó dos polos: el bienestar (salud) y el malestar (enfermedad). Según el autor, no es posible que un organismo vivo logre ninguno de los polos extremos del continuo, es decir, la salud perfecta o el estado completo de enfermedad. Por un lado, toda persona tiene alguna parte insalubre, a pesar de que pueda percibirse a sí mis- 
ma como saludable. Por otro, aún en los estados terminales, mientras haya un soplo de vida, en alguna medida, algunos componentes de la persona se encuentran saludables. De esta forma, el énfasis no debe hacerse en el hecho de que una persona está sana o enferma, sino más bien en qué lugar del continuo se coloca, entre la salud perfecta y el completo estado de enfermedad.

Es precisamente en este continuo de salud-enfermedad donde Antonovsky encuentra puntos comunes con la Teoría General de Sistemas ${ }^{4}$. Concretamente, Antonovsky considera que la salud no es un estado de equilibrio pasivo, sino más bien un proceso inestable, de autorregulación activa y dinámica. El principio básico de la existencia humana no es el equilibrio y la salud, sino el desequilibrio, la enfermedad y el sufrimiento. Es decir, la desorganización y la tendencia hacia la entropía está omnipresente en el organismo humano, como en cualquier otro sistema ${ }^{5}$. En sentido figurado, Antonovsky empleó el concepto de entropía (término prestado de la termodinámica) como una expresión de la tendencia ubicua de los organismos humanos a perder su estructura organizada, así como a la capacidad de reordenarse de nuevo. Aplicado al campo de la salud, esto significa que la salud debe ser constantemente re-creada y que, al mismo tiempo, la pérdida de la salud es un proceso natural y omnipresente, ya que el caos y el estrés, lejos de ser realidades objetivas, son experiencias percibidas, surgidas de demandas internas y/o externas, que forman parte de las condiciones naturales de la vida.

Tal y como explican con detalle Lindstrom y Eriksson, los conceptos fundamentales que Antonovsky desarrolla con esta teoría son los Recursos Generales de Resistencia y el Sentido de Coherencia ${ }^{6}$. Los Recursos Generales de Resistencia (RGRs) son factores biológicos, materiales y psicosociales que hacen más fácil a las personas percibir su vida como coherente, estructurada y comprensible. Los típicos Recursos Generales de Resistencia son el dinero, el conocimiento, la experiencia, la autoestima, los hábitos saludables, el compromiso, el apoyo social, el capital cultural, la inteligencia, las tradiciones y la visión de la vida. Si una persona tiene este tipo de recursos a su disposición o en su entorno inmediato tiene más oportunidades para hacer frente a los desafíos de la vida. Estos recursos ayudan a las personas a construir experiencias coherentes en la vida. Sin embargo, más allá de poseerlos, lo importante es tener la capacidad para utilizarlos, es decir, poseer lo que Antonovsky llamó Sentido de Coherencia (SOC) (Sense of Coherence), convirtiéndose dicho concepto en la clave de su teoría. De hecho, Antonovsky diseñó un cuestionario para medirlo (las diferentes versiones de este cuestionario así como su traducción en varios idiomas se encuentra disponible en la página web del Folkhälsan Research Centre $^{7}$.

Según el autor, el SOC estaría compuesto por tres componentes clave: la comprensibilidad (componente cognitivo), la manejabilidad (componente instrumental o de comportamiento) y la significatividad (componente motivacional). Es decir, por un lado la capacidad del sujeto para comprender cómo está organizada su vida y cómo se sitúa él frente al mundo; por otro, mostrarse capaz de manejarla y, finalmente, sentir que tiene sentido, que la propia vida está orientada hacia metas que se desean alcanzar. Una de las críticas que recibió el modelo estuvo relacionada con la no inclusión de un componente afectivo dentro de este paradigma integrador, estando sobrerrepresentado el aspecto racional o cognitivo ${ }^{8}$. Aunque Antonovsky fue consciente de este problema decidió no extender la teoría para incluir ese cuarto componente.

Un uso óptimo de los Recursos Generales de Resistencia da lugar a que las experiencias vitales se conviertan en promotoras de un nivel alto de SOC, una forma de percibir 
la vida y una capacidad para gestionar exitosamente el infinito número de complejos factores estresantes a los que hay que hacer frente a lo largo de la vida. Es decir, el SOC es la capacidad para percibir que se es capaz de manejar cualquier situación independientemente de lo que está sucediendo en la vida. Antonovsky define el SOC como una orientación global que expresa el grado en que uno tiene una omnipresente y duradera, aunque a la vez dinámica, sensación de confianza, en tres sentidos: (1) los estímulos derivados de los entornos internos y externos en el curso de la vida son estructurados, predecibles y explicables (son comprensibles); (2) los recursos están disponibles para satisfacer las demandas planteadas por los estímulos (son manejables), y (3) estas demandas son retos, dignos de invertirles esfuerzo y compromiso (son significativas) ${ }^{3}$. Lejos de las clásicas estrategias de afrontamiento, el SOC es flexible, no se construye en torno a un conjunto fijo de estrategias a dominar ${ }^{5}$. Es decir, se trataría de un "sexto sentido" útil para la supervivencia, ya que genera habilidades que promueven la salud.

\section{DESARROLLO DEL MODELO SALU- TOGÉNICO: SU INFLUENCIA EN LA PROMOCIÓN DE LA SALUD}

Desde la proclamación de la nueva concepción de salud, realizada en los albores de la constitución de la Organización Mundial de la Salud $^{9}$ hasta la proclamación oficial de la Promoción de la Salud como objetivo de las políticas de salud pública, realizada en la Carta de Ottawa $^{10}$, la atención se fue centrando cada vez más en el desarrollo y potenciación de los recursos de salud para la población. Los principales testigos de este cambio de perspectiva se pueden encontrar en el Informe Lalonde ${ }^{11}$, la Declaración de Alma-Ata, realizada en la Primera Conferencia Internacional sobre Atención Primaria de Salud ${ }^{12}$ o la estrategia "Salud para todos en el año 2000"13. El pilar fundamental de dicho proceso sería el respeto por los Derechos Humanos y la consideración de las personas como participantes activas de su bienestar. En este sentido, los profesionales y las personas se comprometerían mutuamente en un proceso de empoderamiento, de modo que el papel de los profesionales sería apoyar y ofrecer opciones que permitan a las personas tomar decisiones bien fundamentadas, siendo conscientes de los principales determinantes de la salud. Es en la Primera Conferencia Internacional sobre Promoción de la Salud, celebrada en octubre de 1986 en Ottawa (Canadá), cuando la Promoción de la Salud fue definida oficialmente como el proceso que permite a las personas incrementar el control sobre su salud para mejorarla ${ }^{10}$.

En cuanto a la teoría salutogénica, a pesar de no figurar explícitamente en la Carta de Ottawa, ha influido indudablemente en el desarrollo de la Promoción de la Salud. El comienzo de esta influencia se puede decir que sucedió formalmente en 1992, en un seminario celebrado en la Oficina Regional de la OMS en Copenhague, en el que se discutieron las teorías subyacentes en Promoción de Salud. Antonovsky asistió a este taller y presentó su modelo salutogénico como una dirección para la Promoción de la Salud. Entre los acuerdos se concluyó que el enfoque en Promoción de la Salud debía realizarse sobre la salud y no sobre la enfermedad. La orientación salutogénica se presentaba como un paradigma realmente viable para la investigación y la práctica en Promoción de la Salud ${ }^{14}$. En concreto, según resumen Lindstrom y Eriksson, el modelo salutogénico sería una buena base de fundamentación para la Promoción de la Salud, por tres razones principales: en primer lugar, porque el foco se coloca en la resolución de problemas y en la búsqueda de soluciones; en segundo lugar, porque identifica Recursos Generales de Resistencia que ayudan a las personas a moverse en la dirección de salud positiva; $y$, en tercer lugar, porque identifica un sentido o discernimiento global y omnipresente en los individuos, grupos, poblaciones o sistemas, cuya capacidad se plasma en el SOC (comprensión, manejabilidad y significatividad de los acontecimientos vitales) ${ }^{6}$. 
Antonovsky hace uso de una metáfora (el río de la vida) para comparar el punto de vista patogénico con la perspectiva salutogéni$\mathrm{ca}^{3}$. Usando el símil de un río embravecido, el enfoque patogénico considera que las corrientes de ese río representarían el estado de enfermedad, siendo el objetivo rescatar a las personas que se encuentran dentro de ese río, sin tener en cuenta cómo llegaron allí o por qué no son buenos nadadores. Sin embargo, desde una perspectiva de salud pública, habría que reconocer que a veces las personas voluntariamente se tiran al río o se niegan a aprender a nadar. En este sentido y desde una perspectiva salutogénica, Antonovsky prefiere entender que ese río representaría la vida en sí, es decir, un río que da lugar a arroyos con aguas tranquilas o bien a peligrosas corrientes y remolinos. Así, Antonovsky se pregunta cuáles serán las condiciones que determinan que una persona tenga la habilidad de nadar bien, independientemente de la zona del río donde esté. Por lo tanto, el objetivo no se centraría en "salvar" a las personas, sino en conseguir que naden bien, para no ser arrastrados por dicha corriente.

En la representación gráfica del río de la vida (figura 1) presentada por Eriksson y Lindstrom se muestra de manera clara el desarrollo de la medicina (de atención y tratamiento) y de la salud pública (prevención y promoción $)^{15}$. Concretamente, el desarrollo lógico e histórico de la salud pública hacia la Promoción de la Salud presentaría las siguientes cuatro etapas.

En primer lugar, la curación o tratamiento de enfermedades. Según el símil del río, la perspectiva curativa en salud significaría "salvar a la gente de ahogarse", utilizando caras herramientas tecnológicas y profesionales bien entrenados.

En segundo lugar la protección de la salud y prevención de enfermedades. Esta etapa se puede dividir en dos fases:
- Protección. Desde este enfoque, las intervenciones tendrían como objeto evitar los riesgos de caer enfermo, dirigiendo los esfuerzos a la población general, pero otorgándole un papel pasivo en el proceso. Según la metáfora, se trataría de colocar barreras al río para evitar que las personas caigan en él.

- Prevención. En este caso se trataría de prevenir las enfermedades a partir de la intervención activa de las propias personas. Es decir, se trata de administrar un chaleco salvavidas para evitar que las personas se ahoguen en el río.

En la tercera etapa coexistirían la Educación para la Salud y la Promoción de la Salud. Por un lado, desde la Educación para la Salud, los profesionales tratarían de informar, a nivel individual o grupal, de los riesgos para su salud, proporcionando consejos sobre cómo deben vivir para mantener su salud y evitar los riesgos. Progresivamente, con el paso del tiempo, las personas van participando cada vez más en este proceso llegando a ser capaces de tomar sus propias decisiones contando con el apoyo de los profesionales. Volviendo a la metáfora del río, los esfuerzos estarían dirigidos a "enseñar a las personas a nadar". Por otro lado, desde la Promoción de la Salud la responsabilidad de la acción se extendería más allá del sector salud, de modo que la salud se entendería como un derecho humano a conseguir a través de la coordinación de toda la sociedad. En este caso, el individuo se convierte en un sujeto responsable, activo y participante. La tarea de los profesionales sería apoyar y ofrecer opciones para que las personas puedan tomar decisiones bien fundamentadas. La educación sanitaria es aquí sustituida por el aprendizaje acerca de la salud, a partir de la reciprocidad de un diálogo en salud.

Por último, la cuarta etapa se caracterizaría por la mejora de la percepción de la salud, bienestar y calidad de vida. El objetivo último de las actividades de Promoción de la 
Figura 1

Salud en el río de la vida ${ }^{15}$

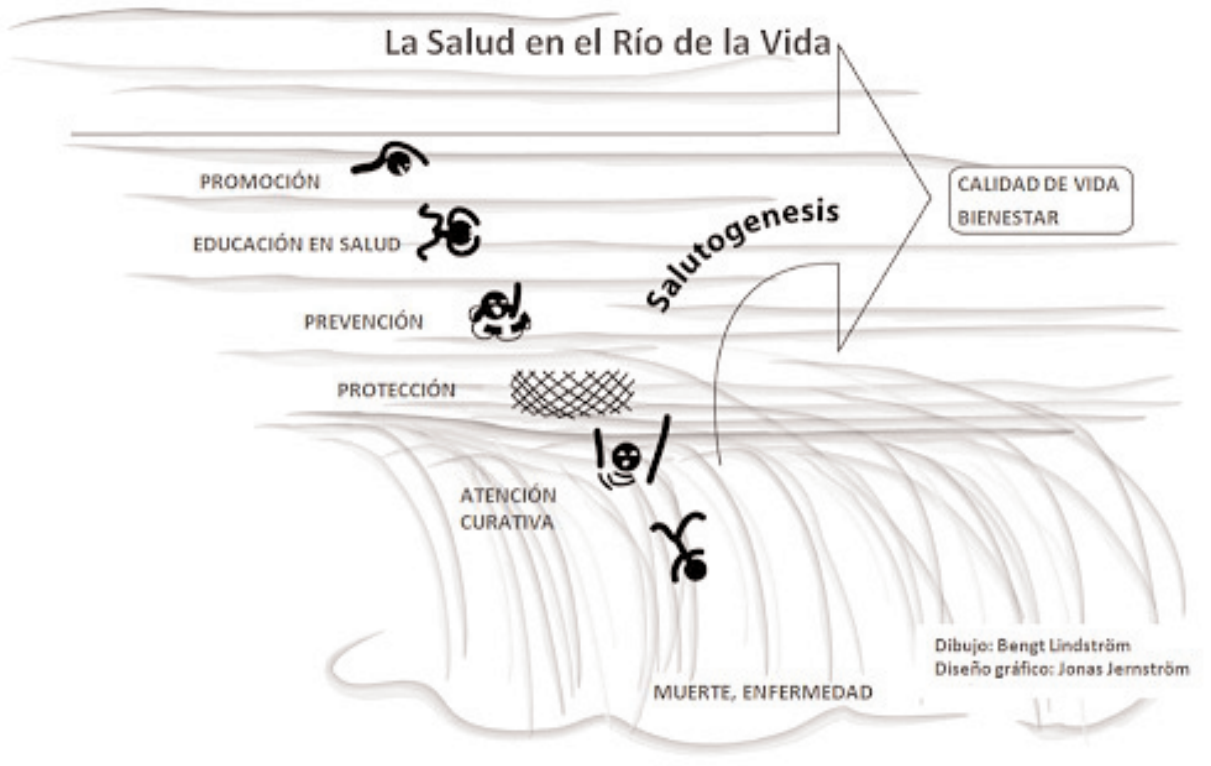

Salud es la creación o facilitación de las condiciones previas necesarias para el desarrollo de una vida saludable. El objetivo último de las actividades de Promoción de la Salud es la creación o facilitación de las condiciones previas necesarias para el desarrollo de una vida saludable; es decir, la clave del proceso está en aprender a reflexionar sobre las opciones que existen en función de las situaciones que ocurren en la vida, sacando partido de aquellas que generan salud, las que mejoran la calidad de vida y las que desarrollan el SOC.

Volviendo a la metáfora desarrollada por Eriksson y Lindstrom, la corriente del río únicamente se entendería, según el modelo biomédico, por su fluir hacia abajo, cuyo fin se encuentra en la cascada. Por tanto, desde esta perspectiva patogénica, únicamente se podría ayudar a las personas que ya tienen problemas para escapar por sí solos de esa corriente que les guía hacia la cascada. Sin embargo, para explicar el cambio de paradigma en el marco salutogénico, la metáfora del río es diferente. En este caso, se trataría del "río de la vida", donde la dirección principal en el fluir del agua ocurre horizontalmente, a pesar de que a lo largo de todo su recorrido existe permanentemente un salto vertical del agua, en forma de cascada. Al nacer, las personas caen en el río y flotan con la corriente. La dirección principal de la vida no es la muerte y la enfermedad, representada por la cascada. Algunas personas nacen cerca del lado opuesto a la cascada, donde pueden flotar con facilidad, las oportunidades de la vida son buenas y tienen muchos recursos a su disposición, como en un estado de bienestar duradero. En cambio, otras personas nacen cerca de la cascada, en el malestar o enfermedad, donde la lucha por la supervivencia es más difícil y el riesgo de estar por encima del borde de la cascada es mucho mayor. El río está lleno de riesgos, pero también de recursos. Sin embargo, el 
resultado de la travesía a través del río de la vida se basa principalmente en la capacidad para identificar y utilizar los recursos necesarios para mejorar las opciones de salud y, en definitiva, la calidad de vida.

\section{RETOS Y EXPANSIÓN DEL MODELO SALUTOGÉNICO EN LA ACTUALIDAD}

A pesar de sus tres décadas de existencia, el modelo salutogénico y, sobre todo, el modelo empírico sustentado en el cuestionario SOC todavía deben hacer frente a una serie de retos.

Para empezar, en varios estudios psicométricos centrados en el SOC se demuestra que el instrumento propuesto por Antonovsky para medirlo tiene adecuados niveles de fiabilidad y validez ${ }^{16-17}$. Sin embargo, es necesario advertir que el cuestionario SOC también ha recibido algunas críticas desde un punto de vista psicométrico, sobre todo por la posibilidad de que este instrumento pueda medir algún constructo más de los señalados en principio por el autor, no estando clara la estructura factorial ${ }^{18-19}$.

En lo que respecta a la propuesta realizada por algunos expertos para utilizar el cuestionario SOC como un instrumento de screening, capaz de identificar a las personas que tienen riesgo de desarrollar un nivel bajo de $\mathrm{SOC}$, es necesario advertir que esta nuevas posibilidades del SOC deben tomarse con cautela. Por un lado, todavía existen problemas para identificar la posición de un individuo en el continuo salud-enfermedad y, por otro lado, hay que tomar en consideración el riesgo que podría suponer el hecho de estigmatizar al grupo de personas que consigan bajas puntuaciones ${ }^{20}$.

Por otro lado, también existe controversia en lo referente a la estabilidad del SOC a lo largo del ciclo vital. Antonovsky asumía que el SOC se desarrollaba hasta los 30 años y a partir de entonces sería relativamente resistente al cambio ${ }^{3}$. Sin embargo, recientemente se están encontrando algunos resultados contradictorios a esa explicación. De hecho, Eriksson y Lindstrom ${ }^{16}$ consideran que el SOC sufre un cierto incremento con la edad durante toda la vida, siendo la estabilidad mayor para las personas con un fuerte SOC $^{21}$.

A pesar de que en sus inicios y a lo largo del siglo XX el modelo no atrajo la atención de la comunidad científica ${ }^{22}$, en el presente la situación está cambiando. Desde 1992 a 2003 se han publicado más de 500 trabajos relacionados con este modelo en todo el mundo y el cuestionario SOC ha sido utilizado en, al menos, 33 lenguas y 32 países diferentes $^{20}$.

En definitiva, teniendo en cuenta estos aspectos, el modelo salutogénico debe seguir estudiándose ya que muchas de sus nociones aún necesitan aclararse, probarse y posicionarse con respecto a otros conceptos y teorías ${ }^{23}$. Por ejemplo, muy recientemente, Lundman et al. han demostrado la existencia de un factor relacionado con la "fuerza interior" 24 , que se encuentra presente tanto en el modelo salutogénico como en otras teorías y conceptos afines, como son la resiliencia ${ }^{25-}$ ${ }^{26}$, la personalidad resistente ${ }^{27}$, el sentido de la vida ${ }^{28}$, la auto-trascendencia ${ }^{29}$, las teorías de la base segura ${ }^{30} \mathrm{o}$ las teorías del aprendizaje significativo ${ }^{31}$.

En España, diferentes grupos de investigación han usado el cuestionario SOC, estudiando su relación con algunos de esos conceptos. El primer estudio publicado en España sobre el SOC data de 1997 y aborda la relación entre salud y una serie de variables de personalidad, como son el sentido de coherencia, la personalidad resistente y la autoestima $^{32}$. Este trabajo se enfoca en el papel del sentido de coherencia como moderador de salud ante circunstancias estresantes, traduciendo y adaptando al español el cuestionario SOC en su versión de 29 ítems. 
Este trabajo ha tenido el mérito de informar acerca de las características psicométricas del SOC en población española por vez primera, ofreciendo indicadores psicométricos semejantes a los ofrecidos por adaptaciones a poblaciones normalizadas en otros paí$\operatorname{ses}^{20}$.

Dos años más tarde se publicó un nuevo trabajo, liderado también por el doctor Moreno-Jiménez, en el que utilizó la misma versión del cuestionario. En dicho artículo se aborda nuevamente el papel del SOC como mediador entre el estrés y la salud, pero en esta ocasión se centra en el proceso de burnout sufrido por una muestra de profesores de educación primaria ${ }^{33}$. Los datos de fiabilidad para esta muestra de nuevo se encuentran entre los parámetros habituales para este cuestionario.

Tras estos dos artículos, no se volvió a divulgar en España datos sobre el sentido de coherencia hasta 2007, cuando se publicó un artículo sobre la validación transcultural del cuestionario SOC en su versión reducida (SOC-13) en una muestra de personas mayores de 70 años ${ }^{21}$. Este estudio se centró en el proceso de validación y propiedades psicométricas del cuestionario, ofreciéndose nuevamente datos óptimos en fiabilidad y nuevos aportes de validez convergente del SOC, con medidas autorreferidas de salud física, calidad de vida y depresión para la población anciana.

Por último, en 2008, un equipo de investigación de la Universidad de Oviedo abordó, en pacientes diagnosticados de fibromialgia, la relación entre la salud, por un lado, y las dimensiones de personalidad y el sentido de coherencia por otro ${ }^{34}$. Entre otros resultados, se encontró un nivel más bajo de sentido de coherencia (con niveles inferiores en las dimensiones de comprensibilidad y significatividad) en los pacientes con fibromialgia en comparación con el grupo control, caracterizado por no presentar ninguna patología crónica. Sin embargo, en este caso, los auto- res no hacen referencia a si el cuestionario SOC utilizado proviene de una traducción anteriormente validada, por lo que se puede asumir que usaron su propia versión, aunque no ofrecieron datos psicométricos al respecto.

Para terminar, un potencial del modelo salutogénico es el relacionado con sus implicaciones prácticas $^{35}$. Es evidente que este modelo es un buen enfoque para ser aplicado en la construcción de políticas y programas de salud pública, así como en otras políticas también relacionadas con la salud. Es decir, es posible reorientar y revitalizar la Promoción de la Salud hacia modelos de acción basados en los activos -assets- para la Salud $^{36-37}$; no solo pensando en los servicios de salud, sino en la potenciación de los Recursos Generales de Resistencia para cada persona y comunidad. En este sentido, son destacables los trabajos de Kretzman y Mcknight sobre la utilización de los mapas de activos para la salud de la comunidad, es decir, el uso de las fortalezas que la comunidad tiene para descubrir capacidades y talentos individuales, colectivos y ambientales existentes en el contexto ${ }^{38}$.

Estas nuevas líneas en el modelo salutogénico se han plasmado en diferentes iniciativas de políticas de salud pública de alcance. Así, por ejemplo, en el marco de la Presidencia Española de la Unión Europea, centrada en la consecución de la equidad en salud $^{39}$, y dentro de su programa de seminarios, conferencias y congresos, se llevaron a cabo dos eventos para los que la fílosofía del modelo salutogénico ha tenido especial relevancia.

Por un lado, desde el 28 al 30 de abril del 2010, se celebró en Sevilla el simposio Health Assets for Young People's Wellbeing, organizado por el equipo español del estudio Health Behaviour in School-aged Children (de la Universidad de Sevilla), la Universidad de Hertfordshire (Reino Unido), la Organización Mundial de la Salud y el 
Ministerio de Sanidad y Política Social de España. El simposio reunió a expertos de todo el mundo en torno a los temas de la salutogénesis, la resiliencia y el capital social. En concreto, y en lo que respecta a la salutogénesis, se contó con la participación de Monica Eriksson y Bengt Lindstrom, referentes actuales en el estudio de la salutogénesis, quienes trataron de centrar la aplicación del constructo a las poblaciones de adolescentes y jóvenes. El objetivo general de este simposio fue el de contribuir al avance de los enfoques basados en activos (y no sólo en carencias o déficits) para mejorar el bienestar de los jóvenes, poniendo de relieve la aportación que los investigadores, los responsables políticos y los profesionales pueden hacer en ese proceso. En definitiva, se trató de un simposio centrado en las implicaciones prácticas del modelo de activos propuesto, entre otros, por Antony Morgan y Erio Ziglio ${ }^{36,40}$, y que cuenta entre sus principios básicos con la teoría salutogénica de Antonovsky.

Por otro lado, centrado en una etapa posterior de la vida, el 28 y 29 de junio del 2010 tuvo lugar en Madrid la conferencia Mental Health and Well-being in Older People Making it Happen, organizada conjuntamente por la Comisión Europea de Sanidad y Consumo y el Ministerio de Sanidad y Política Social de España. En ella los expertos apoyaron la idea de utilizar el enfoque salutogénico en las actividades y políticas de Promoción de la Salud para las personas mayores. En este sentido, en una revisión de literatura que cumple la función de documento base de la conferencia, los autores explicaron cómo el SOC ha sido útil para identificar los factores personales y ambientales que son potencialmente protectores de la salud mental positiva en la vejez ${ }^{41}$. Es decir, se profundizó en la relación entre el concepto de envejecimiento exitoso y la salutogénesis, así como la autoestima, la autoeficacia y el sentido de control sobre la vida. De igual manera, se propuso aplicar los principios salutogénicos dentro de la prácti- ca profesional, teniendo en cuenta especialmente el ámbito de los cuidados paliativos y el trabajo con las familias de personas mayores recién fallecidas, así como el estrés y los conflictos en el entorno profesional.

En conclusión, estos dos eventos son un buen ejemplo de los esfuerzos que se están realizando actualmente en España para aplicar los principios salutogénicos a la investigación y a la práctica sanitaria. Además, merece la pena advertir que, curiosamente, los dos ejemplos que se han citado se corresponden con aplicaciones de la teoría salutogénica a dos etapas evolutivas bien diferentes (adolescencia y vejez), y que históricamente han sido objeto de estudio por sus deficiencias, riesgos y problemas. Este nuevo enfoque permite ahora analizarlas e intervenir sobre ellas desde la perspectiva del desarrollo positivo y de los activos con los que la persona y sus circunstancias cuentan. Quizá en breve podamos estar hablando de los resultados de la aplicación de este nuevo enfoque en salud pública, lo que habrá significado con toda seguridad entrar en un nivel de orden superior en la prevención de la enfermedad y en la mejora de la calidad de vida de los ciudadanos.

\section{BIBLIOGRAFÍA}

1. Antonovsky A, Maoz B, Dowty N., Wijsenbeek H. Twenty-five years later: A limited study of the sequelae of the concentration camp experience. Soc Psychiatry Psychiatr Epidemiol. 1971; 6: 186-93.

2. Antonovsky A. Health, Stress and Coping. San Francisco: Jossey-Bass; 1979.

3. Antonovsky A. Unraveling the Mystery of Health. How People Manage Stress and Stay Well. San Francisco: Jossey-Bass; 1987.

4. von Bertalanffy L. General System theory: Foundations, Development, Applications. New York: George Braziller; 1969.

5. Antonovsky A. Complexity, conflict, chaos, coherence, coercion and civility. Soc Sci Med. 1993; 37 : 969-81. 
6. Lindstrom B, Eriksson M. Contextualizing salutogenesis and Antonovsky in public health development. Health Promot Int. 2006; 21: 238-44.

7. Health Promotion Research Team, Folkhälsan Research Centre. SOC questionnaire [citado 20 de enero 2010]. Disponible en:

http://www.salutogenesis.fi/eng/SOC_questionnaire.19.html.

8. Sigerist J. Sense of coherence and sociology of emotions. Soc Sci Med. 1993; 37, 978-79.

9. World Health Organization. WHO Constitution. Genova: World Health Organization; 1948.

10. Organización Mundial de la Salud. Carta de Ottawa para la promoción de la salud. Conferencia Internacional sobre la Promoción de la Salud: Hacia un nuevo concepto de la Salud Pública. Ottawa: Salud y Bienestar Social de Canadá, Asociación Canadiense de Salud Pública; 1986.

11. Lalonde M. A New Perspective on the Health of Canadians. Ottawa: Information Canada; 1974.

12. Organización Mundial de la Salud. Atención primaria de la salud. Informe de la Conferencia Internacional sobre Atención Primaria de Salud Alma- Ata. Ginebra: OMS; 1978.

13. World Health Organization. Global Strategy for Health for All by the Year 2000. Genova: World Health Organization; 1981.

14. Antonovsky A. The salutogenic model as a theory to guide health promotion. Health Promot Int. 1996; 11: 11-18.

15. Eriksson M, Lindstrom B. A salutogenic interpretation on the Ottawa Charter. Health Promot Int. 2008; 23 : 190-98.

16. Eriksson M, Lindstrom B. Validity of Antonovsky's sense of coherence scale: a systematic review. J Epidemiol Community Health. 2005; 59: 460-66.

17. Feldt T, Lintula H, Suominen S, Koskenvuo M, Vahtera J, Kivimäki M. Structural validity and temporal stability of the 13-item sense of coherence scale: Prospective evidence from the population-based HeSSup study. Qual Life Res. 2006; 16: 483-93.

18. Feldt T, Rasku A. The structure of Antonovsky's Orientation to Life Questionnaire. Pers Individ Dif. 1998; 25: 505-16.

19. Larsson G, Kallenberg K. Dimensional analysis of sense of coherence using structural equation modelling. Eur J Pers. 1999; 13: 51-61.

20. Eriksson M. Unraveling the mystery of salutogenesis. The evidence base of the salutogenic research as measured by Antonovsky's Sense of Coherence Scale [tesis doctoral]. Turku: Åbo Akademi University Vasa; 2007.

21. Virues-Ortega J, Martinez-Martin P, del Barrio JL, Lozano LM. Validación transcultural de la Escala de Sentido de Coherencia de Antonovsky (OLQ-13) en ancianos mayores de 70 anos. Med Clin (Barc). 2007; 128: 486-92.

22. Bengel J, Strittmatter R, Willmann H. What keeps people healthy? The current state of discussion and the relevance of Antonovsky's salutogenic model of health. Cologne: Federal Centre for Health Education; 1999.

23. Eriksson M, Lindström B. Bringing it all together the salutogenic response to some of the most pertinent public health dilemmas. En: Morgan A, Ziglio E, Davies M, editores. Health Assets in a Global Context. New York: Springer; 2010.

24. Lundman B, Aléx L, Jonsén E, Norberg A, Nygren B, Santamäki Fischer R et al. Inner strength- A theoretical analysis of salutogenic concepts. Int J Nurs Stud. 2010; 47: 251-60.

25. Luthar SS, Cushing G. Measurement issues in the empirical study of resilience: An overview. En: Glantz MD, Johnson JL, editores. Resilience and developmental- Positive life adaptations. Nueva York: Kluwer Academic/Plenum Press; 1999.

26. Rutter M. Psychosocial resilience and protective mechanisms. Am J Orthopsychiatry. 1987; 57: 316-31.

27. Kobasa SC. Stressful life events, personality, and health: an inquire into hardiness. J Pers Soc Psychol. 1979; 37: 1-9.

28. Frankl VE. Logotherapy and existential analysis- a review. Am J Psychother, 1966; 20: 252-60.

29. Reed PG. Toward a nursing theory of self-transcendence: Deductive reformulation using developmental theories. ANS Adv Nurs Sci. 1991; 13: 64-77.

30. Bowlby J. Apego y pérdida. Barcelona: Paidós Ibérica; 1998.

31. Piaget J. Los estadíos en la psicología del niño. Buenos Aires: Nueva Visión; 1971.

32. Moreno-Jiménez B, Alonso M, Álvarez. Sentido de 
la coherencia, personalidad resistente, autoestima y salud. Rev Psicol Salud.1997; 9: 115-37.

33. Moreno-Jiménez B, Gonzalez JL, Garrosa E. Burnout docente, sentido de la coherencia y salud percibida. Rev Psicopatol Psicol Clín. 1999; 4: 163-80.

34. Besteiro JA, Lemos S, Muñiz J, Costas C, Weruaga A. Dimensiones de personalidad, sentido de coherencia y salud percibida en pacientes con un síndrome fibromiálgico. Int J Clin Health Psychol. 2008; 8: 411-27.

35. Lindström B, Eriksson M. A salutogenic approach to tackling health inequalities. En: Morgan A, Ziglio E, Davies M, editores. Health Assets in a Global Context: Theory, Methods, Action. New York: Springer; 2010.

36. Morgan A, Ziglio E. Revitalising the evidence base for public health: An assets model. Promotion \& Education. 2007; 14: 17-22.

37. Hernán M, Lineros C. Los activos para la salud. Promoción de la salud en contextos personales, familiares y sociales. Revista Fundesfam. 2010; 2: Disponible en: http://www.fundesfam.org/REVISTA\%20FUNDESFAM $\% 201 / 007$ revisiones.htm

38. Kretzman JP, Mcknight JL. Building Communities from the Inside Out: a Path Toward Finding and Mobilizing a Community Assets. Chicago: ACTA Publications; 1993.

39. Ministerio de Sanidad y Política Social. Hacia la Equidad en Salud: Monitorización de los determinantes sociales de la salud y reducción de las desigualdades en salud. Informe independiente de expertos comisionado por la Presidencia Española de la UE. Madrid: Ministerio de Sanidad y Política Social; 2010.

40. Morgan A, Davies M, Ziglio E. Health Assets in a Global Context: Theory, Methods, Action. New York: Springer; 2010.

41. Billings J, Hashem F. Literature Review - Salutogenesis and the Promotion of Positive Mental Health in Older People. EU Thematic conference "Mental Health and Well-being in Older People - Making it Happen". Madrid: European Commission Directorate-General for Health and Consumers and the Spanish Ministry of Health and Social Affairs; 2009 [citado 4 de agosto 2010]. Disponible en:

http://ec.europa.eu/health/mental_health/docs/older_sa lutogenesis.pdf. 